
Pseudomonas aeruginosa-induced

SUBJECT AREAS: MEDICAL RESEARCH

INNATE IMMUNITY

BACTERIA

BIOLOGICAL SCIENCES

Received

26 April 2011

Accepted

11 July 2011

Published

8 August 2011

Correspondence and requests for materials should be addressed to M.G. (mgadjeva@rics. bwh.harvard.edu) \section{ocular infection}

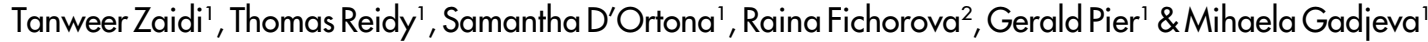

'Department of Medicine, Channing Laboratory, Brigham and Women's Hospital, Harvard Medical School, Boston MA 02115 , 2Laboratory of Genital Tract Biology, Department of Obstetrics, Gynecology and Reproductive Biology, Brigham and Women's Hospital, Harvard Medical School, Boston, MA 02115.

Eye trauma and contact lens wear are the main factors that predispose to the development of infectious keratitis. The existing therapies fail to control the inflammation-driven tissue damage that occurs during Pseudomonas aeruginosa infection. Antibiotic treatment reduces bacterial burdens, but better interventions are needed to alleviate tissue damage resulting from local inflammation. We have previously documented that inhibition of macrophage migration inhibitory factor (MIF) reduces the bacterial levels and the inflammatory damage during keratitis. Here, we report that mice deficient for CD74, the putative MIF receptor, developed milder Pseudomonas aeruginosa-induced disease, characterized by decreased proinflammatory mediators and reduced bacterial presence in the cornea. However, topical inhibition of MIF using antibodies applied to the cornea further promoted recovery from disease, suggesting that in addition to MIF-dependent signaling events, MIF-triggered CD74-independent signaling pathways regulate sensitization to $P$. aeruginosa-induced infection.

E ye trauma and contact lens wear are the main factors that predispose to the development of infectious keratitis. In the United States, one in 2,500 daily contact lens wearers and one in 500 overnight wearers develop bacterial keratitis each year. Corneal disease ranges from self-limiting to sight threatening, the latter requiring rapid diagnosis and treatment to prevent vision loss ${ }^{1}$. The organism most often isolated from contact lens associated corneal ulcers is Pseudomonas aeruginosa ${ }^{2}$. Pseudomonas keratitis is a rapidly developing disease that can progress to involve the entire cornea within 48 hours, which may lead to corneal perforation. The existing therapies often fail to control the excessive tissue damage that is induced during P. aeruginosa infection ${ }^{3}$. While antibiotic treatment can reduce the bacterial burden, tissue damage occurs as a result of exaggerated local inflammation. Thus, a combined therapy including agents that control local inflammatory responses could be highly beneficial.

As in any human infectious disease, inflammatory responses in microbial keratitis are both beneficial and harmful. Initially inflammatory mediators are needed to recruit polymorphonuclear neutrophils (PMNs) and effectively resolve infection ${ }^{4}$. However, if innate immunity elicits an overly-robust response, inflammatory mediators become a part of a hypersensitivity response, which contributes to tissue damage and increases pathology $y^{5,6}$. Using model of bacterial keratitis in mice induced by scratch injury, typical of corneal trauma, we previously demonstrated that deficiency in macrophage migration inhibitory factor (MIF) appears protective during acute infection induced by $P$. aeruginosa as evidenced by reduced corneal pathology and increased bacterial clearance when a small molecular inhibitor of MIF was used for treatment during infection ${ }^{7}$. Hence, a better understanding of MIF-driven processes in response to infection will pinpoint key molecular targets for development of innovative therapeutic strategies.

MIF is an integral component of inflammatory responses ${ }^{8}$. MIF directly, or indirectly sustains expression of a large panel of pro-inflammatory cytokines such as TNF $-\alpha$, IFN- $\gamma$, IL-1 $\beta$, IL-2, IL-6, IL-8, MIP-2, NO, COX2, products of the arachidonic acid pathway, matrix metalloproteinases, etc ${ }^{9-12}$. The majority of these processes most likely depend on the interaction of MIF with a receptor complex composed of CD74/CD44, the former component being primarily studied as the major histocompatibility complex (MHC) class II invariant chain and the later component noted for its ability to bind hyaluronic acid and other matrix metalloproteinases ${ }^{13}$. Binding of MIF to CD74/CD44 results in activation of Mitogen-Activated Protein Kinase (MAPK), production of PGE2 ${ }^{14}$ and further induction of inflammatory mediators ${ }^{14-16}$. About $8-10 \%$ of total cellular CD74 is expressed on the cell 
surface complexed with $\mathrm{CD} 44^{16}$, suggesting that CD74 may have important non-chaperone -related functions when complexed with CD44. Consistently, blockade of CD74 reduces MIF-dependent monocyte arrest ex vivo, chemokine expression, and neutrophil recruitment ${ }^{17}$.

To examine the role of CD74 in $P$. aeruginosa-induced ocular keratitis, CD74 KO and C57BL6 control mice were infected with different laboratory strains or clinical isolates of $P$. aeruginosa and disease pathology examined. As expected, CD74 KO mice had milder disease progression with decreased proinflammatory mediators released such as IL- $1 \beta$ and TNF- $\alpha$, and reduced bacterial presence in the cornea. However, topical inhibition of MIF by application of specific antibody onto the cornea promoted further recovery from the disease, suggesting that in addition to previously described MIFinduced CD74-dependent pathways, sensitization to disease occurs via MIF-triggered CD74-independent pathways.

\section{Results}

Bacterial burdens after Pseudomonas aeruginosa eye infection are elevated in WT mice compared with CD74 KO mice. CD74 KO and C57BL6 WT mice were infected with different doses of the invasive $P$. aeruginosa strain 6294 with corneal pathology and bacterial burdens determined at different times post-infection (Figure 1). Forty-eight $\mathrm{h}$ after infection with $1 \times 10^{5} \mathrm{cfu} /$ eye of $P$. aeruginosa strain 6294 the differences in bacterial loads between the CD74 KO and C57BL6 mice were significant (Figure 1A): there were about 1000 -fold fewer bacteria recovered from the corneal tissue of the infected CD74 KO mice compared to the infected C57BL6 mice. Consistent with the reduced bacterial levels, the CD74 KO mice infected with $P$. aeruginosa strain 6294 had a significant decrease in corneal pathology $(\mathrm{P}=0.01)$ as evident by haematoxylin-eosin staining of tissue sections obtained from the eyes of the infected animals (Figure 1B). To determine whether the reduced pathology in the CD74 KO mice was evident following a higher bacterial challenge dose, separate cohorts of CD74 KO and C57BL6 WT mice were infected with a fifty-fold higher dose of $P$. aeruginosa $6294\left(5 \times 10^{6} \mathrm{cfu} /\right.$ mouse eye; Figure 1C). Under these conditions, the infected CD74 KO mice also exhibited milder disease with significantly fewer bacteria recovered from the infected corneas (Figure 1C). As expected, the pathology was not as radically reduced as when the lower bacterial inoculum was used to induce infection. As is known from published data ${ }^{18}$, if the infection is left to proceed for a longer period, perforation of the cornea will occur within 5 days in the C57BL6 mouse strain that is highly sensitive to $P$. aeruginosa keratitis. To determine whether the infected CD74 $\mathrm{KO}$ were capable of maintaining the milder phenotype, a separate cohort of mice were infected with P,aeruginosa 6294 and corneal pathology monitored. At $72 \mathrm{~h}$ post-infection, the sensitive C57BL6 presented with significant corneal opacity, whereas the infected CD74 KO mice appeared to control the infection better (Figure 1D). Consistently, lower bacterial counts were recovered from the infected corneas of the CD74 KO mice.

To determine whether the reduced pathology in CD74 KO mice was specific to the $P$. aeruginosa 6294 strain, similar experiments were performed using another invasive $P$. aeruginosa strain, $\mathrm{PAO} 1$ (Figure 2A and B), as well as a cytotoxic strain, 6077 (Figure 2C and $\mathrm{D})$. In both cases, the bacterial levels recovered from the corneal tissue were lower in the infected CD74 $\mathrm{KO}$ when compared to the infected C57BL6 mice as early as $48 \mathrm{~h}$ post-infection. Significant reduction in the pathology scores in the CD74 knockout mice was evident at $72 \mathrm{~h}$ post-infection, consistent with the observations for the $P$. aeruginosa strain 6294 .

CD74 regulates inflammatory responses to $P$. aeruginosa. Since CD74 deficiency impairs MHCII-driven antigen presentation, it is possible that the activation of T-cell responses to $P$. aeruginosa could be altered in the CD74 KO mice ${ }^{19,20}$. Therefore, the observed lower bacterial burdens and, in some cases, milder disease in the CD74 KO may be due to alterations of the $\mathrm{T}$-cell activation profile in addition to MIF-dependent activities. To examine the inflammatory cytokine profiles in the infected CD74 KO and C57BL6 control mice, cohorts of mice were inoculated with $P$. aeruginosa 6294 and a multiplex cytokine assay performed on corneal samples at $48 \mathrm{~h}$ and $72 \mathrm{~h}$ post-infection. At $48 \mathrm{~h}$ after infection there were no significant differences in the levels of TNF- $\alpha, \mathrm{KC}$, IL- $1 \beta$, or IFN- $\gamma$ between C57BL6 and CD74 KO mice (Figure 3). In addition, no differences were measured in IL-2, IL-4, IL-5, and IL-10 (Figure 4). However, at $72 \mathrm{~h}$ post-infection TNF- $\alpha$, and IL- $1 \beta$ were modestly,

A
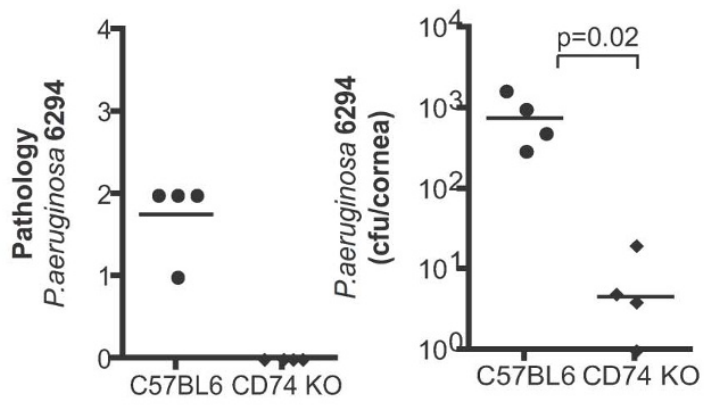

B

Central cornea

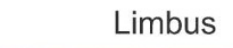

C57BL6
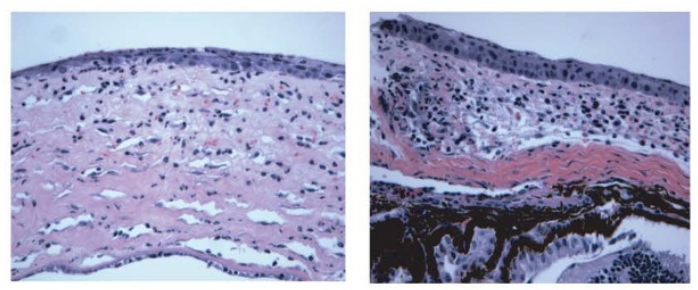

CD74 KO
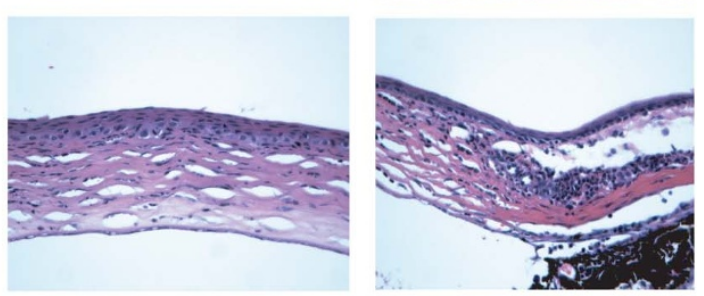

C
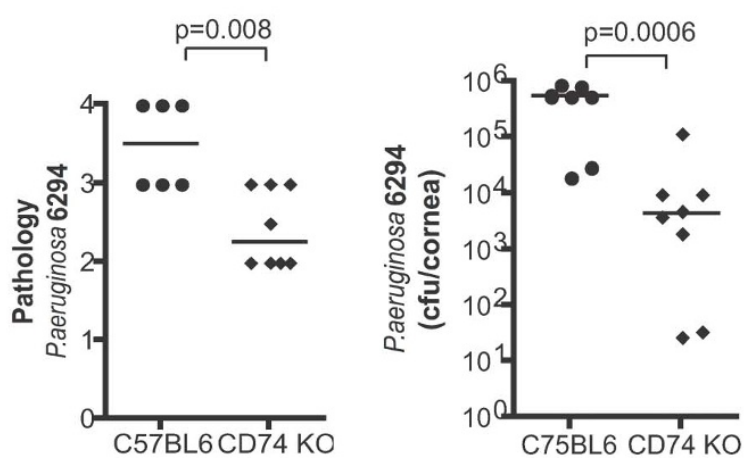

Figure $1 \mid$ CD74 KO mice have milder $P$. aeruginosa-induced ocular keratitis. (A) Pathology and levels of $P$. aeruginosa strain 6294, determined $48 \mathrm{~h}$ following infection with $1 \times 10^{5} \mathrm{cfu}$ onto eyes of CD74 KO and WT C57BL6 mice. Points indicate values for individual mice, bars the medians. Data are from a representative experiment out of two performed. (B) Histopathology images of corneal sections from C57BL6 and CD74 KO mice. (C) and (D) Levels and pathology of P. aeruginosa strain 6294, determined at $72 \mathrm{~h}$ following infection with $5 \times 10^{6} \mathrm{cfu}$ onto eyes of CD74 KO and WT C57BL6 mice. Points indicate values for individual mice, bars the medians. Data are from a representative experiment out of three performed. 


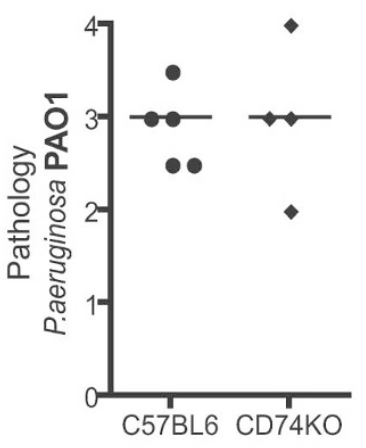

B

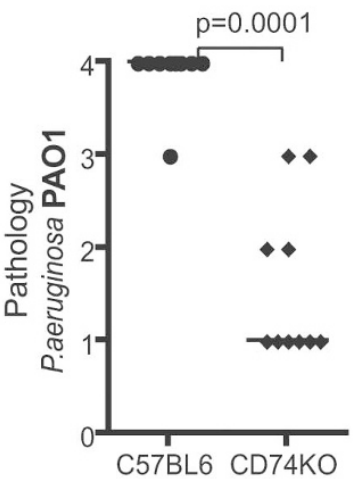

C

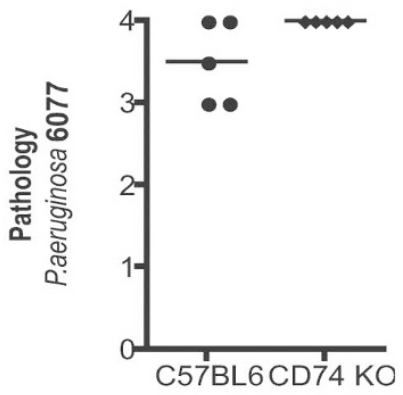

D
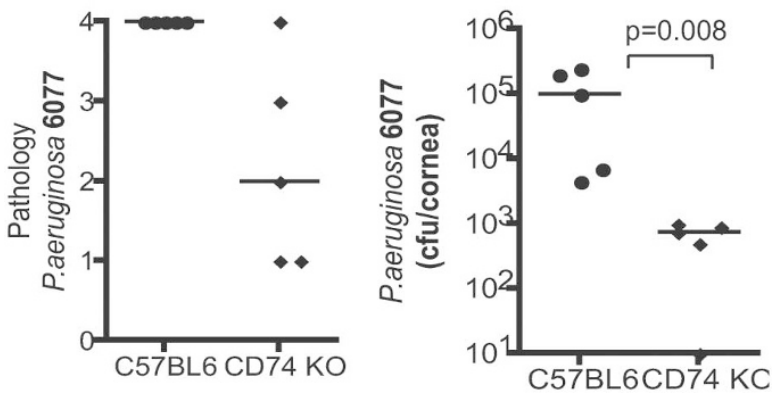

Figure $2 \mid \mathrm{CD} 74 \mathrm{KO}$ mice exhibit reduced bacterial burdens in the cornea after infection with PAO1 and 6077 strains. (A) and (B) Pathology and levels of $P$. aeruginosa strain PAO1, determined at $48 \mathrm{~h}(\mathrm{~A})$ and $72 \mathrm{~h}(\mathrm{~B})$ following infection with $5 \times 10^{6}$ cfu onto eyes of CD74 KO and WT C57BL6 mice. Points indicate values for individual mice, bars the medians. Data are from a representative experiment out of two performed. (C) and (D) Pathology and levels of P. aeruginosa strain 6077, determined at $48 \mathrm{~h}(\mathrm{~A})$ and at $72 \mathrm{~h}(\mathrm{~B})$ following infection with $5 \times 10^{5} \mathrm{cfu}$ onto eyes of CD74 $\mathrm{KO}$ and WT C57BL6 mice. Points indicate values for individual mice, bars the medians. Data are from a representative experiment out of three performed.

but significantly decreased in the CD74 KO mice, whereas IFN- $\gamma$ showed the opposite tendency. IFN- $\gamma$ was significantly increased in the infected CD74 KO mice, when compared to the infected C57BL6 mice (Figure 3). There appeared to be a tendency for moderately elevated KC and IL-12, but it did not reach significance when the two groups of mice were analyzed.

CD74 KO mice recover from $P$. aeruginosa-induced keratitis after local application of antibodies to MIF. We have previously demonstrated that MIF KO mice had reduced bacterial levels and pathology from $P$. aeruginosa-induced ocular infection ${ }^{21}$. In contrast, the CD74 KO mice did not manifest the same degree of differences nor spontaneously recovered from disease, except when a low dose of $P$. aeruginosa was used to induce infection. These observations suggested that MIF-induced responses are not completely coordinated with CD74-dependent responses and, therefore, it is likely that MIF induces responses to $P$. aeruginosa infection through an alternate means such as use of receptors in addition to CD74. To test this hypothesis experimentally, antibody to MIF was produced in rabbits and used to treat infected cohorts of CD74 KO and C57BL6 mice after the onset of keratitis.

Five $\mu$ of the rMIF antisera were applied onto the eyes of the infected mice every $8 \mathrm{~h}$ after the onset of infection. The CD74 KO mice treated with the rMIF antisera exhibited a significant reduction in the corneal cfu and in the corneal pathology score compared to mice receiving normal rabbit antiserum (NRS) (Figure 5A). To determine whether the combined administration of antibiotics and antisera to MIF promoted better disease recovery, CD74 KO and C57BL6 mice were infected with the gentamicin sensitive strain $P$. aeruginosa 6294 and treated with gentamicin in combination with either antiMIF antisera or NRS control antisera (Figures 5B and 5C). Both the WT and CD74 KO mice treated with the combination of gentamicin and antisera to rMIF had lower bacterial burdens and corneal pathology compared to controls given gentamicin and NRS, and the CD74 $\mathrm{KO}$ mice given the combination treatment had no evidence of pathology or detectable bacterial infection when euthanized $48 \mathrm{~h}$ after infection.

\section{Discussion}

In the eye, inflammation is strictly regulated to preserve delicate ocular tissues ${ }^{22}$. However, ocular infections can trigger intense inflammation raising important question: can controlled inhibition of inflammation be therapeutically beneficial during infection? We have demonstrated that inhibition of a key amplifier of inflammation - MIF - is therapeutically beneficial to control P. aeruginosa-induced eye infection ${ }^{7}$. Therefore, gaining insight into MIF-dependent signaling events is crucial for design of appropriate interventional therapies. Here, we analyzed the role of CD74, the putative MIF receptor, in $P$. aeruginosa-induced pathology by characterizing ocular disease progression in the CD74 $\mathrm{KO}$ mice and C57BL6 control mice. Deficiency for CD74 appeared protective during $P$. aeruginosainduced corneal damage resulting generally in milder pathology or reduced tissue bacterial levels in the infected CD74 KO mice when compared to control mice. However, the protection was not comparable to what was previously observed in infected MIF KO mice, suggesting that MIF-triggered CD74-independent pathways, as well as a MIF-independent effects from loss of CD74, could contribute to the pathologic response to acute $P$. aeruginosa keratitis in the absence of this molecule.

Several functions of CD74 distinct from MIF-induced signaling could contribute to the observed phenotype in the CD74 KO mice. $\mathrm{CD} 74$ functions as chaperone for $\mathrm{MHC}$ loading and regulates $\mathrm{T}$ cell maturation by affecting both the positive and negative selection of naive CD4 $+\mathrm{T}$ cells. Consequently, CD74 deficiency impairs MHCII-driven antigen presentation, resulting in changes in the $\mathrm{CD} 4+\mathrm{T}$ cell maturation. However, CD74 $\mathrm{KO}$ mice are not devoid of $\mathrm{CD} 4+\mathrm{T}$ cells, instead they have a reduced frequency of $\mathrm{CD} 4+\mathrm{T}$ cells $^{19}$. It also appears that the CD4 $+\mathrm{T}$ cells from the CD74 KO mice proliferate normally in vitro and respond normally to immunization with protein antigens. Furthermore, delayed type hypersensitivity 
A
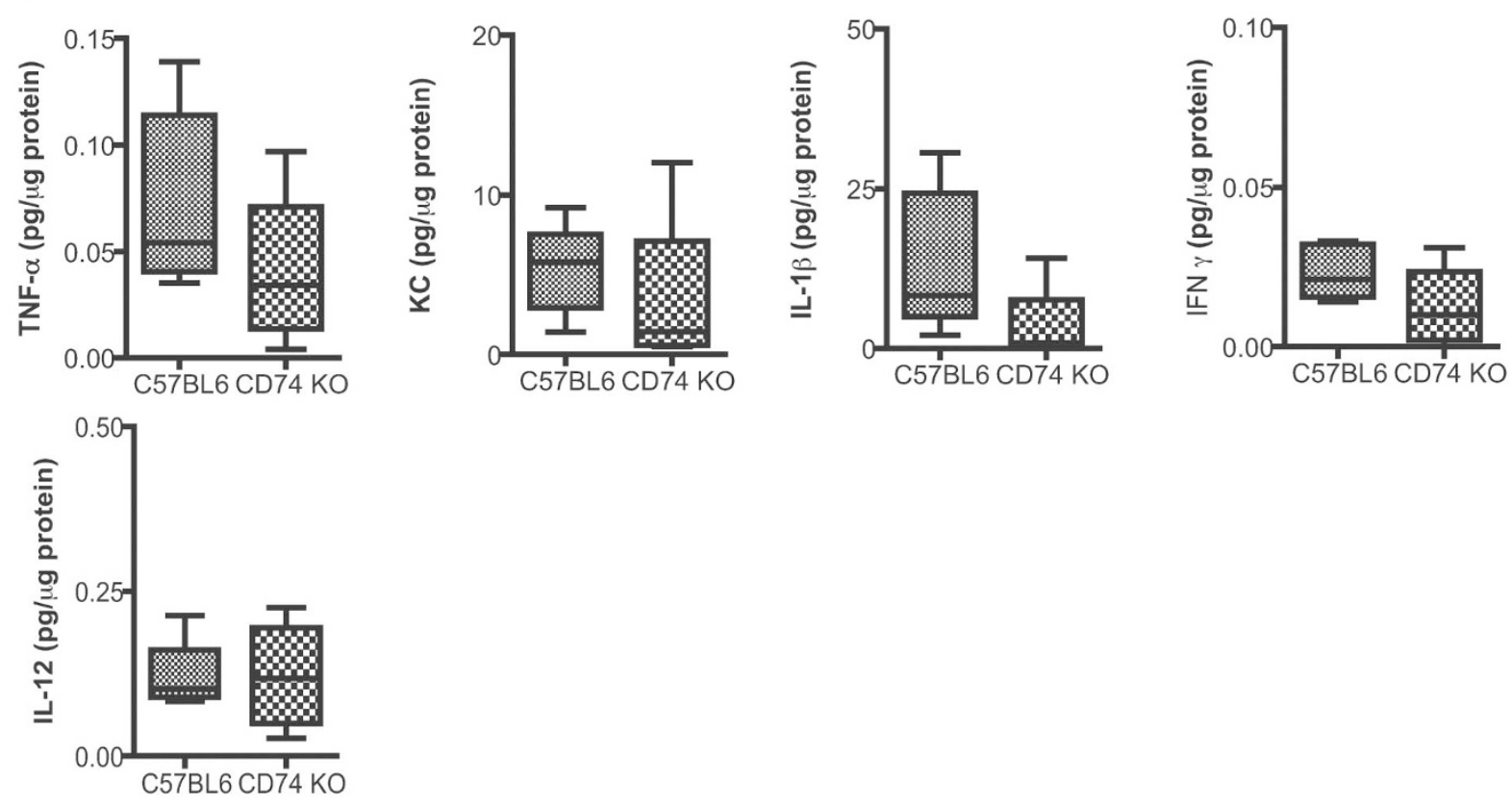

B
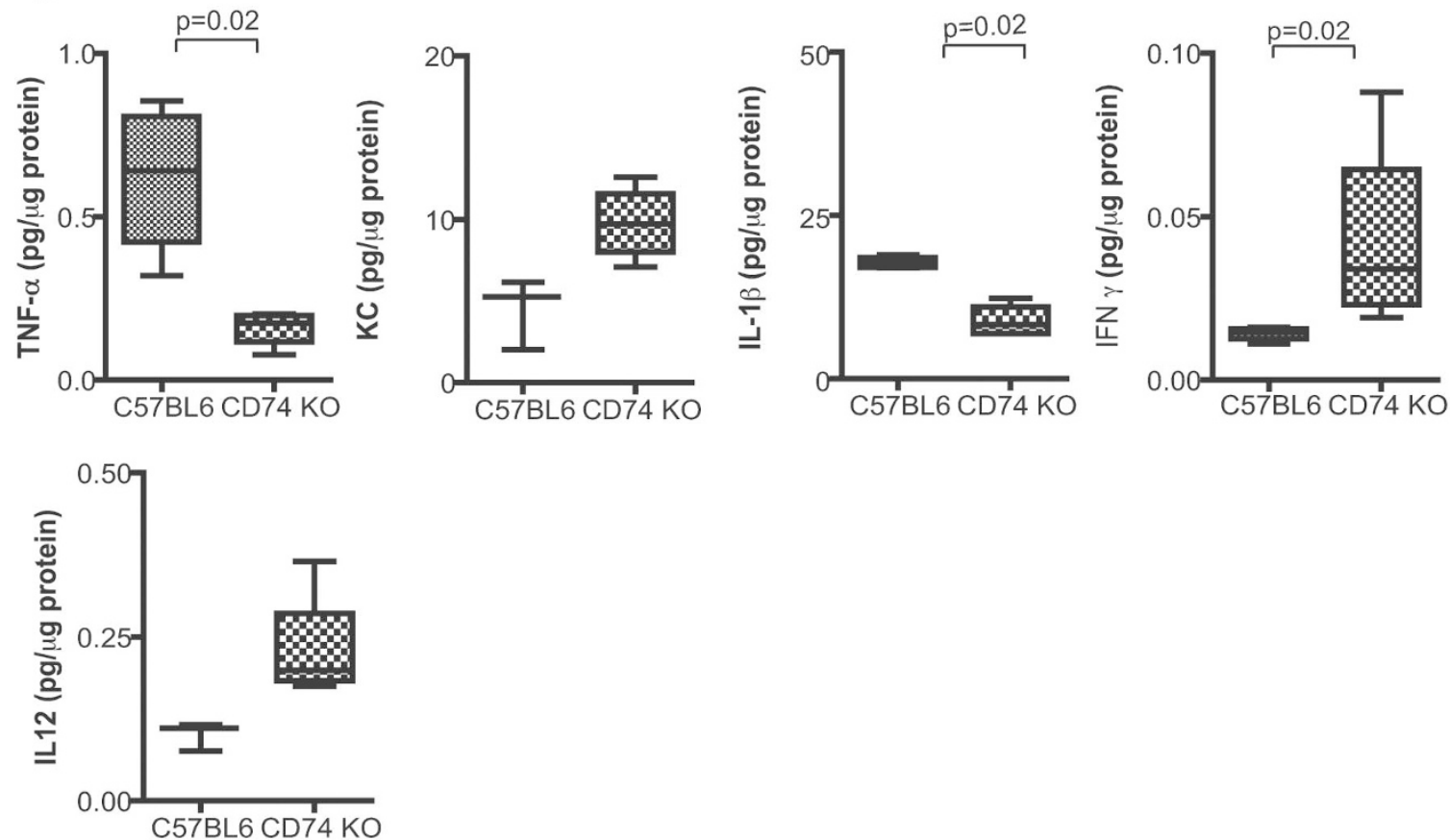

Figure $3 \mid \mathrm{CD} 74$ regulates proinflammatory responses to $P$. aeruginosa. Groups of 5 individual CD74 KO mice and 5 C57Bl6 mice were infected with $5 \times 10^{6} \mathrm{cfu}$ placed onto scratch-injured eyes. Mouse corneas were harvested at $48 \mathrm{~h} \mathrm{(A)}$ and $72 \mathrm{~h}(\mathrm{~B})$ after infection. The levels of mouse cytokines in corneal lysates were simultaneously measured using a MSD multiplex 7-spot electrochemiluminescence (ECL) assay. The levels of cytokines measured in the different groups were compared with the Mann-Whitney U test.

(DTH) responses appear comparable with that of the C57BL6 control mice, suggesting that Th1 skewing is occurring ${ }^{20}$. The ability of the naïve $\mathrm{CD} 4+\mathrm{T}$ cells in the $\mathrm{CD} 74 \mathrm{KO}$ mouse environment to develop into Th1 or, possibly Th17, subsets was verified in a colitis model wherein disease was induced by administration of 2,4,6-trinitrobenzenesulfonic acid (TNBS). TNBS challenged CD74 KO and C57BL6 mice had comparable inflammatory responses ${ }^{20}$. Interestingly, Topilski et al reported that the CD74 KO mice mounted slightly elevated IFN $-\gamma$ responses after antigenic challenge, suggestive of elevated Th1 responses ${ }^{20}$. Consistent with these findings, we do not find defects in Th1 inflammatory cytokine synthesis in response to $P$. aeruginosa corneal challenge. The Th1 versus Th2 profiling was examined based on relative presence of IL-2, IL-4, IL-5, IL-10, IL-12, IFN- $\gamma$ and TNF- $\alpha$ in the corneal tissues. While the IL-2, IL-12 levels were comparable in the infected CD74 KO and C57BL6 mice, the IFN- $\gamma$ levels were slightly elevated in the CD74 KO, when compared to the P. aeruginosa-infected C57BL6 mice. Therefore, we ruled out that the milder phenotype observed in the CD74 KO was due to significant decrease in disease-mediating Th1 skewing ${ }^{23}$.

The genetic background of the mice influences disease severity in ocular keratitis $^{24}$. While C57BL6 mice appear sensitive, BALB C mice have milder responses to $P$. aeruginosa-induced keratitis. Therefore, 

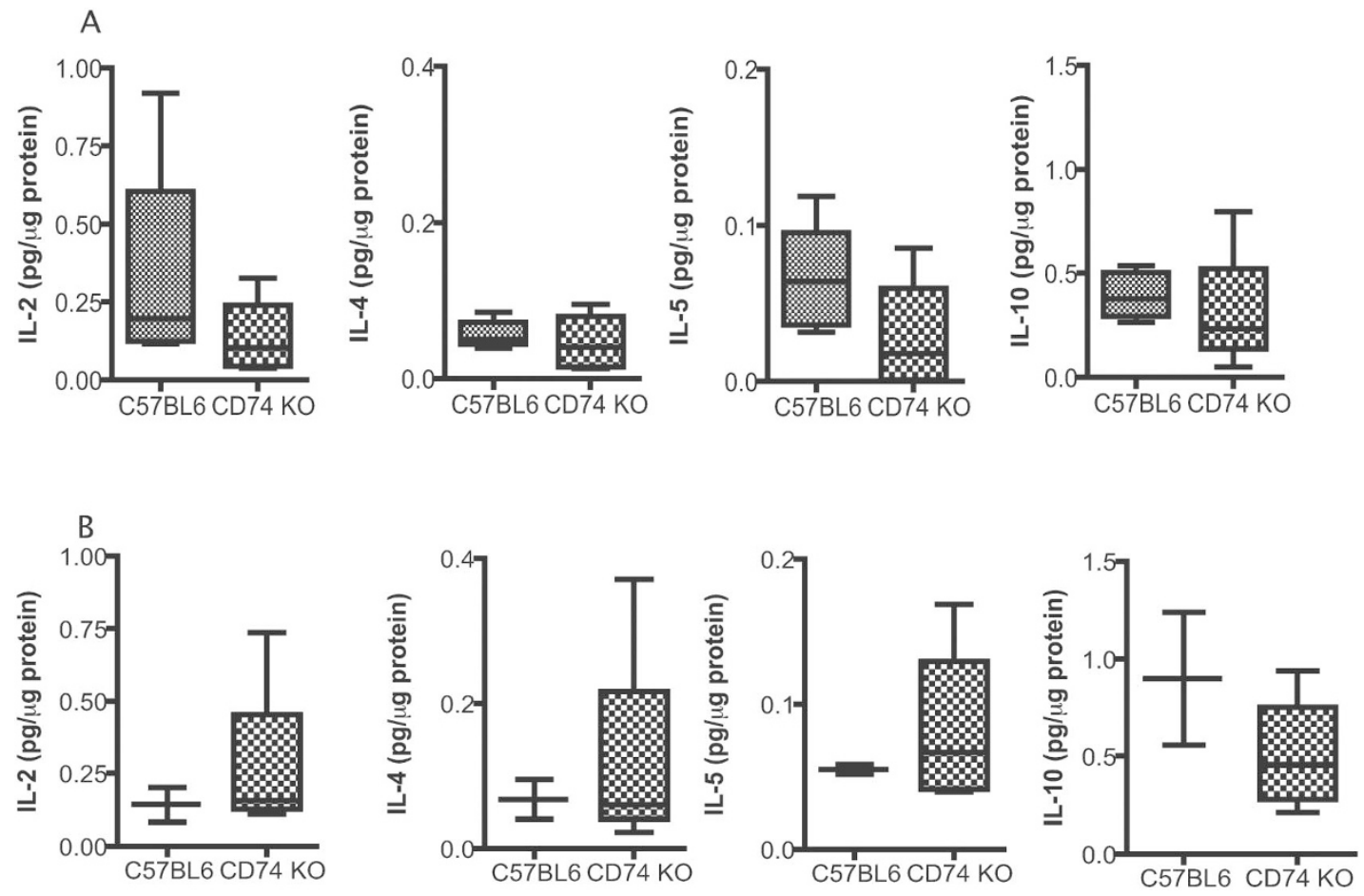

Figure $4 \mid \mathrm{CD} 74$ deficiency does not radically inhibit Th2 cytokines present in the cornea after $\boldsymbol{P}$. aeruginosa infection. Groups of $5 \mathrm{CD} 74 \mathrm{KO}$ mice and $5 \mathrm{C} 57 \mathrm{Bl} 6$ mice were infected with $5 \times 10^{6} \mathrm{cfu}$ placed onto scratch-injured eyes. Mouse corneas were harvested at $48 \mathrm{~h}(\mathrm{~A})$ and $72 \mathrm{~h}$ (B) after infection. The levels of mouse cytokines in corneal lysates were simultaneously measured using a MSD multiplex 7-spot electrochemiluminescence (ECL) assay. The levels of cytokines measured in the different groups were compared with the Mann-Whitney U test.

a possible defect in Th2 skewing in the CD74 KO mice could affect disease progression in these animals. To address this issue, Th2 subset specific cytokines were quantified and no significant changes in the IL-4 and IL-5 levels were observed, suggesting no major alterations in the CD4+ Th2 subset skewing (Figure 4). These data are consistent with previously reported observations that the CD74 KO mice develop normal Th2 responses ${ }^{25}$.

Previous reports have highlighted the importance of MIF-induced CD74 regulated inflammatory responses during autoimmune inflammation ${ }^{14,16}$. MIF binding to the CD74/CD44 complex results in elevated MAPK activation and sustained cytokine production by macrophages and/or epithelial cells ${ }^{7,12,26,27}$. Our previous data suggested that protection against $P$. aeruginosa-induced acute keratitis correlated with decreased TNF- $\alpha$, IL- 6 , and IL-1 $\beta$ recovered from the infected corneas ${ }^{7}$. Consistently, we observed lower levels of TNF- $\alpha$ and IL- $1 \beta$ at $72 \mathrm{~h}$ post-infection, but not at $48 \mathrm{~h}$ post-infection, in $\mathrm{CD} 74 \mathrm{KO}$ mice, suggesting that a reduction in the sustained inflammatory responses may contribute to some of the lessened disease observed in these mice. However, as these differences were not manifest early in the infection they likely reflect the effects from reduced bacterial levels recovered from the CD74 KO mice, but these reductions probably came too late to have an impact on the initial development of inflammation-induced corneal damage during P. aeruginosa infection.

The conclusion that there are CD74-independent cellular response pathways that are induced by MIF supported by the observation that when the $\mathrm{CD} 74 \mathrm{KO}$ mice were therapeutically treated with antisera to MIF after the onset of disease there were further reductions in corneal pathology and bacterial burdens, consistent with previously published data ${ }^{21}$ that inhibiting MIF biologically reduced disease pathology in the infected C57BL6 mice. Future studies should establish whether these functions of MIF could be attributed to its ability to serve as a non-cognate ligand for CXCR2 ${ }^{28}$ or relate to cell-intrinsic functions of MIF mediated by MIF-interacting intracellular proteins ${ }^{29}$.
In conclusion, infection can negatively affect host fitness through two distinct mechanisms. Firstly, $P$. aeruginosa can directly cause tissue damage through a variety of virulence activities. Secondly, tissue damage can be caused by the inflammatory responses to the pathogen. Thus, treatment of infection must come in two forms to enable recovery from the two sources of tissue damage. Our data shows that combined with antibiotic treatment, the antibody to MIF markedly reduced pathology and bacterial levels in WT C57BL6 mice, but essentially resulted in complete resolution of pathology and bacterial infection in the CD74 KO mice. These findings suggest that a combination of antibiotics, inhibition of MIF and inhibition of CD74-dependent signaling could provide an optimal therapeutic strategy for treating $P$. aeruginosa infections.

\section{Methods}

Mice. All studies were performed in accordance with the Harvard Medical School Animal Care and Use Committee guidelines. Breeding pairs of CD74 knockout (KO) mice were a kind gift from Dr. F. Vascotto (Harvard Medical School). Control mice (C57BL6) were obtained from Taconic Farms. Mice were housed and bred in the Channing Laboratory Animal Care Facilities

Bacterial strains and inocula. Invasive $P$. aeruginosa strains PAO1, 6294 and Exotoxin U-producing cytotoxic strain 6077 were used throughout these experiments. Effects from low dose $\left(1 \times 10^{5} \mathrm{cfu} / \mathrm{eye}\right)$ and high dose $\left(5 \times 10^{6} \mathrm{cfu} /\right.$ eye $)$ inocula were initially evaluated to determine the doses to use in the in vivo infection studies. Generally, the bacterial strains were grown overnight at $37^{\circ} \mathrm{C}$ on tryptic soy broth agar plates and bacterial cells suspended to a concentration of $1 \times 10^{9} \mathrm{cfu} / \mathrm{ml}$ in saline solution.

Purification of rMIF. The human MIF cDNA containing IMAGE clone (ATCC, VA) was amplified by PCR, sequenced and subcloned into E. coli BL21 expression pet11b plasmid as in $^{30}$. Recombinant protein was expressed in E. coli BL21 cells grown overnight at $37^{\circ} \mathrm{C}$ on lysogeny (L) agar plates containing $50 \mu \mathrm{g}$ carbenicillin $/ \mathrm{mL}$ (Sigma-Aldrich, MO). Individual colonies were selected and grown in $2 \mathrm{ml} \mathrm{LB}$ media containing $50 \mathrm{ug} / \mathrm{ml}$ carbenicillin with shaking for $2 \mathrm{~h}$ at $37 \mathrm{C} .1-2 \mathrm{~mL}$ of the bacterial cultures were transferred to $50 \mathrm{~mL}$ conical tubes each containing $25 \mathrm{~mL}$ LB with $50 \mu \mathrm{g} / \mathrm{ml}$ carbinicillin (Sigma-Aldrich, MO). Individual colonies were selected and grown with shaking for $2 \mathrm{~h}$ at $37^{\circ} \mathrm{C}$ in $2 \mathrm{ml}$ lysogeny broth (LB) media containing $50 \mu \mathrm{g}$ carbenicillin $/ \mathrm{ml}$. One to two $\mathrm{ml}$ of the bacterial cultures were transferred to $50 \mathrm{ml}$ conical tubes each containing $25 \mathrm{ml}$ LB with $50 \mu \mathrm{g}$ carbenicillin $/ \mathrm{ml}$. The 

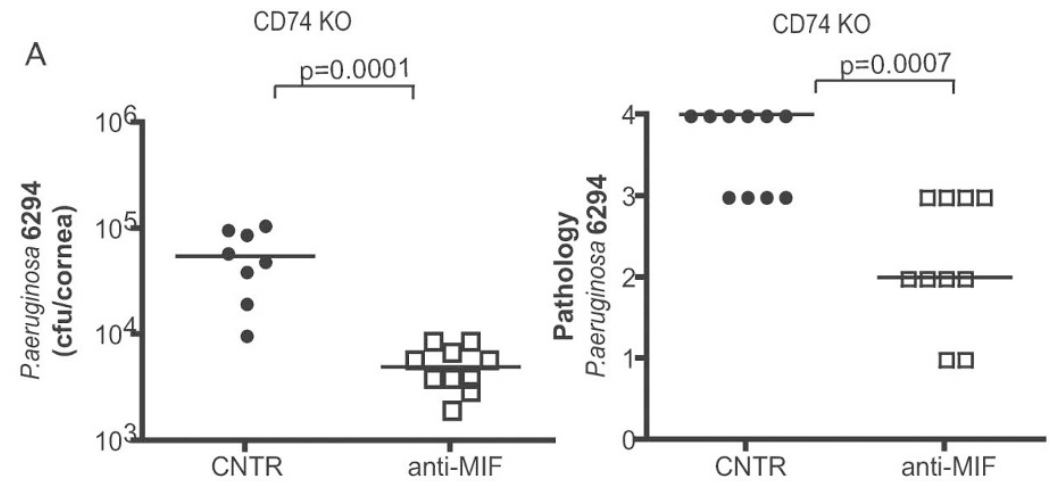

B

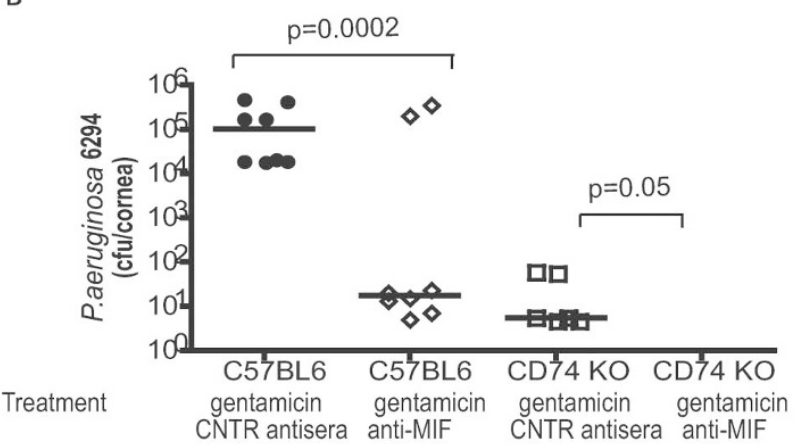

C

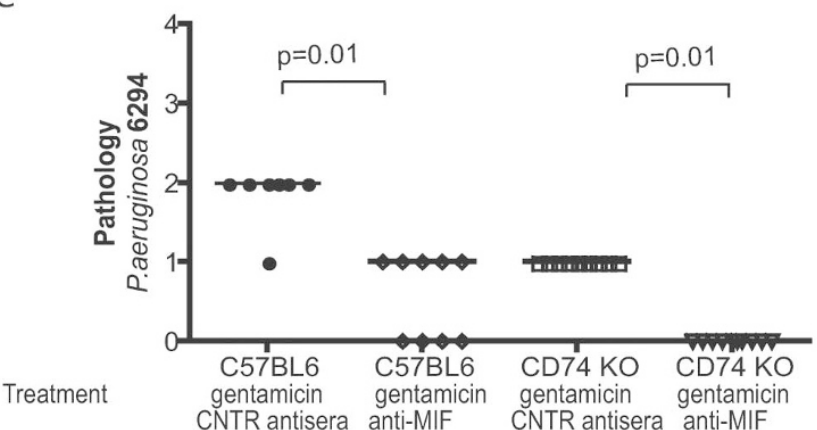

Figure $5 \mid$ Treatment with anti-MIF antisera promotes further recovery from $P$. aeruginosa-induced corneal infection and pathology in CD74 KO mice. (A) Groups of CD74 KO mice were treated with either rabbit control antisera or anti-MIF antisera every $8 \mathrm{~h}$ after the induction of infection for the duration of 2 days after which infected corneas were harvested, homogenized, and bacteria quantified. Points indicate values from individual mice, bars the medians. (B) Groups of C57BL6 and CD74 KO mice were treated with either gentamicin, non-immune rabbit antisera, or gentamicin and anti-MIF antisera every 8 hours after infection for 2 days after which infected corneas were harvested, and bacteria quantified. (C) Pathology scores plotted. Points indicate values from individual mice, bars the medians. Data analysis performed with 1way Anova with Dunn's Multiple Comparison Test (Prizm 4.0). Each experiment was repeated twice.

cultures were shaken for several hours untill the bacterial cultures reached an $\mathrm{OD}_{600}>0.8$. About $10 \mathrm{ml}$ of bacterial culture was transferred to Erlenmeyer flasks containing $500 \mathrm{~mL} \mathrm{LB}$ with $50 \mu \mathrm{g}$ carbenicillin/ml and the bacterial culture density adjusted to an $\mathrm{OD}_{600} 0.1-0.2$. Cultures were incubated with shaking at $37^{\circ} \mathrm{C}$ for $1.5 \mathrm{hrs}$, the $\mathrm{OD}_{600}$ was measured every $30 \mathrm{~min}$. When the cultures reached an $\mathrm{OD}_{600} \sim 0.9,1 \mathrm{mM}$ isopropyl $\beta$-D-1-thiogalactopyranoside (IPTG) (Invitrogen, MD) was added to induce transcription of the cloned MIF gene and the E. coli cells grown for additional 3-3.5 hrs with shaking at $37^{\circ} \mathrm{C}$. Bacteria were harvested by centrifugation at $3000 \mathrm{rpm}$ for 15 min using Beckman ultracentrifuge. The bacterial pellets were stored at $-20^{\circ} \mathrm{C}$

E.coli pellets were lysed with glass beads (Sigma-Aldrich, MO) in 25 mM HEPES, $1 \%$ Triton X-100, 1 mM EDTA, pH 7.5 buffer. The lysate was centrifuged in a Beckman refrigerated $(4 \mathrm{C})$ centrifuge at $10000 \mathrm{~g}$ for 20 minutes and passed through $47 \mathrm{~mm} 5$ micron filters, followed by $0.45 \mathrm{u}$ filter using a vacuum filtration setup. Approximately $175 \mathrm{~mL}$ of clarified rMIF E. coli lysate, harvested from $500 \mathrm{ml}$ of bacterial cultures, was loaded onto a $42 \mathrm{~mL}$ Fractogel DEAE anion exchange column in $25 \mathrm{mM}$ HEPES, $0.1 \%$ Tween 20 , pH 7.5 buffer at $13 \mathrm{ml} /$ minute. The effluent and the wash were pooled. The $\mathrm{pH}$ of the pooled material was adjusted with $30 \mathrm{~mL}$ of 0.5 M MES, pH 5.5. The resultant solution had a pH between 5.5 and 6.0. This material was loaded onto a CM Sepharose Cation Exchange column and eluted with a step gradient at $0.5 \mathrm{M}$ MES, $500 \mathrm{mM} \mathrm{NaCl}, \mathrm{pH}$ 5.5. Purified rMIF was

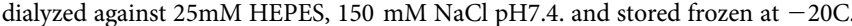

Generation of the rabbit polyclonal anti-MIF antisera. rMIF containing liposomes were prepared for immunization purposes. The liposome composition was $25 \mathrm{mg} / \mathrm{mL}$ lipids, $0.25 \mathrm{mg} / \mathrm{mL} \mathrm{CpG}, 1 \mathrm{mg} / \mathrm{mL} \mathrm{rMIF}$. The lipids were formulated as a blend of $40 \%$ phosphatidyletanolamine (15:0) (Avanti Polar Lipids, AL; $663 \mathrm{~g} / \mathrm{mole}$ ), 40\% phopshatidylcholine (15:0) (Avanti Polar Lipids, AL; $705 \mathrm{~g} / \mathrm{mole}$ ), 10\% Cholesterol (Avanti Polar Lipids, AL; 386 g/mole), 10\% DC-Cholesterol (Avanti Polar Lipids, AL; $537 \mathrm{~g} / \mathrm{mole}$ ). Rabbits were immunized in the popliteal lymph node with $100 \mu \mathrm{g} \mathrm{rMIF/} \mathrm{liposome} \mathrm{mixture,} \mathrm{rested} \mathrm{for} \mathrm{three} \mathrm{weeks,} \mathrm{and} \mathrm{then} \mathrm{re-challenged}$ again in the popliteal lymph node. After 4 weeks of rest, rabbits were immunized intramuscularly with $100 \mu \mathrm{g} \mathrm{rMIF} / \mathrm{liposomes}$ and sera were harvested a week later. The immunizations were carried out at Lampire Biological Laboratories and endpoint binding titers of 12,800 and 25, 600 obtained in two different rabbits.

Infection model. Infections were carried out as described previously ${ }^{31}$. Briefly, mice were anesthetized with injections of $100 \mathrm{mg}$ ketamine $/ \mathrm{Kg}$ and $10 \mathrm{mg}$ xylazine $/ \mathrm{Kg}$. Three $0.5 \mathrm{~cm}$ scratches were made on the cornea and an inoculum of $P$. aeruginosa delivered in $5 \mu \mathrm{l}$ onto the eye. Mice remained sedated for about $30 \mathrm{~min}$. For evaluation of corneal pathology, daily scores are recorded by an observer unaware of the experimental status of the animals based on a scoring system using a graded scale of 0 to 4 as follows: 0 , eye macroscopically identical to the uninfected contra-lateral control eye; 1 , faint opacity partially covering the pupil; 2 , dense opacity covering the pupil; 3 , dense opacity covering the entire anterior segment; and 4, perforation of the 
cornea, phthisis bulbi, shrinkage of the globe after inflammatory disease, or both. To determine the levels of bacteria in the cornea 48 or $72 \mathrm{~h}$ after infection, mice were sacrificed, eyes enucleated and corneas dissected from the ocular surface. To quantify the levels of $P$. aeruginosa, corneas were excised and suspended in a tryptic soy broth, $0.5 \%$ Triton X-100 solution, vortexed, serial dilutions made and plated on $P$. aeruginosa selective cetrimide plates.

For treatment, groups of mice were infected with $P$. aeruginosa strain 6294 , then treated topically for 2 days with $5 \mu \mathrm{l}$ of a solution of $100 \mu \mathrm{g}$ gentamicin/ml (Invitrogen, MD), along with a rabbit polyclonal antisera to MIF. Anti-sera and gentamycin treatments were administered every 8 hours after the initiation of infection. Bacteria in the cornea were quantified 2 days after infection by homogenizing and plating the corneal tissue. Separate cohorts of CD74 KO mice were treated with either the antiserum to MIF or a pre-immune control antiserum in the absence of gentamicin. All antisera were pre-adsorbed with $P$. aeruginosa to eliminate any natural anti-bacterial reactivity.

Histopathology examinations. Eyes were enucleated from euthanized mice and fixed in $4 \%$ paraformaldehyde then embedded in paraffin. Four mm sections were cut, and stained with hematoxylin-eosin to visualize tissue morphology following previously used techniques ${ }^{32}$.

Preparation of corneal lysates. Groups of 7 MIF KO mice and 7 C57BL6 mice were infected with $1 \times 10^{6} \mathrm{P}$. aeruginosa strain $6294 \mathrm{cfu}$ placed onto scratch-injured eyes. Mouse corneas were harvested at $48 \mathrm{~h}$ and $72 \mathrm{~h}$ after infection, washed in F12 media, then each cornea was homogenized in $500 \mu \mathrm{l}$ PBS containing a mix of protease inhibitors (Complete, Roche) and supplemented with $0.5 \%$ Triton to disrupt plasma membranes using an OMNI International tissue master homogenizer 125. Solutions were clarified by 5 min centrifugation at $4 \mathrm{C}$ and maximum rpm in a table-top Eppendorf 5415R centrifuge. The supernatants were stored frozen at $-80^{\circ} \mathrm{C}$ until processed for cytokine analysis.

Cytokine analysis. Mouse cytokines in corneal lysates were measured using a Meso Scale Discovery (MSD) multiplex 7-spot electrochemiluminescence (ECL) assay and outputs measured by an ultra low noise charge-coupled device (CCD) Imager 2400 (Meso Scale Discovery, Gaithersburg, MD, USA). The cytokines measured included interleukin (IL-1, TNF-a, IL-2, IL-4, IL-5, IL-10, IFN- $\gamma$ to establish Th1 versus Th2 profiling in the tissues. The MSD ECL platform has been previously validated against cytokine standards recommended by WHO and U.K. National Institute for Biological Standards and Control (NIBSC) and by comparison to traditional ELISA ${ }^{33}$. Cytokine concentrations were adjusted to total protein levels measured in the corneal extract by the BCA assay (Thermo Scientific, Rockford, IL) using a multi-label Victor 2 counter to measure colorimetric reactions (Perkin Elmer, Boston, MA).

Statistical analysis. Statistical analysis of the differences in the cytokine levels were performed by Mann- Whitney $U$ tests and differences were considered significant if the $\mathrm{p}$ value was $<0.05$ (Prism 4.0 for Macintosh). Statistical analysis of the corneal pathology scores was either by use of the Mann-Whitney U test for pair-wise comparisons or the Kruskal-Wallis non-parametric ANOVA with Dunn's Procedure for Multigroup comparisons and individual 2-group comparisons (Prism 4.0 for Macintosh).

1. Robertson, D. M., Petroll, W. M., Jester, J. V., \& Cavanagh, H. D. Current concepts: contact lens related Pseudomonas keratitis. Cont Lens Anterior Eye 30, 94-107 (2007).

2. Fleiszig, S. M. \& Evans, D. J. The pathogenesis of bacterial keratitis: studies with Pseudomonas aeruginosa. Clin Exp Optom 85, 271-278 (2002).

3. Hazlett, L. D. \& Hendricks, R. L. Reviews for immune privilege in the year 2010: immune privilege and infection. Ocul Immunol Inflamm 18, 237-243.

4. Sun, Y. et al. TLR4 and TLR5 on corneal macrophages regulate Pseudomonas aeruginosa keratitis by signaling through MyD88-dependent and -independent pathways. J Immunol 185, 4272-4283.

5. Thakur, A., Barrett, R. P., Hobden, J. A., \& Hazlett, L. D. Caspase-1 inhibitor reduces severity of pseudomonas aeruginosa keratitis in mice. Investigative ophthalmology \& visual science 45, 3177-3184 (2004).

6. Medzhitov, R. Damage control in host-pathogen interactions. Proceedings of the National Academy of Sciences of the United States of America 106, 15525-15526 (2009).

7. Gadjeva, M., Nagashima, J., Zaidi, T., Mitchell, R. A., \& Pier, G. B. Inhibition of macrophage migration inhibitory factor ameliorates ocular Pseudomonas aeruginosa-induced keratitis. PLoS Pathog 6, e1000826.

8. Flaster, H., Bernhagen, J., Calandra, T., \& Bucala, R. The macrophage migration inhibitory factor-glucocorticoid dyad: regulation of inflammation and immunity. Molecular endocrinology (Baltimore, Md) 21, 1267-1280 (2007).

9. Bernhagen, J. et al. MIF is a pituitary-derived cytokine that potentiates lethal endotoxaemia. Nature 365, 756-759 (1993).

10. Bernhagen, J., Calandra, T., \& Bucala, R. The emerging role of MIF in septic shock and infection. Biotherapy 8, 123-127 (1994).
11. Calandra, T., Bernhagen, J., Mitchell, R. A., \& Bucala, R. The macrophage is an important and previously unrecognized source of macrophage migration inhibitory factor. The Journal of experimental medicine 179, 1895-1902 (1994).

12. Calandra, T. et al. MIF as a glucocorticoid-induced modulator of cytokine production. Nature 377, 68-71 (1995).

13. Shachar, I. \& Haran, M. The secret second life of an innocent chaperone: the story of CD74 and B cell/chronic lymphocytic leukemia cell survival. Leuk Lymphoma.

14. Leng, L. et al. MIF signal transduction initiated by binding to CD74. The Journal of experimental medicine 197, 1467-1476 (2003).

15. Lue, H. et al. Rapid and transient activation of the ERK MAPK signalling pathway by macrophage migration inhibitory factor (MIF) and dependence on JAB1/ CSN5 and Src kinase activity. Cell Signal 18, 688-703 (2006).

16. Shi, X. et al. CD44 is the signaling component of the macrophage migration inhibitory factor-CD74 receptor complex. Immunity 25, 595-606 (2006).

17. Schwartz, V. et al. A functional heteromeric MIF receptor formed by CD74 and CXCR4. FEBS letters 583, 2749-2757 (2009).

18. Hazlett, L. D. Inflammatory response to Pseudomonas aeruginosa keratitis. The ocular surface 3 (4 Suppl), S139-141 (2005).

19. Elliott, E. A. et al. The invariant chain is required for intracellular transport and function of major histocompatibility complex class II molecules. The Journal of experimental medicine 179, 681-694 (1994).

20. Topilski, I., Harmelin, A., Flavell, R. A., Levo, Y., \& Shachar, I. Preferential Th1 immune response in invariant chain-deficient mice. J Immunol 168, 1610-1617 (2002).

21. Gadjeva, M., Nagashima, J., Zaidi, T., Mitchell, R. A., \& Pier, G. B. Inhibition of macrophage migration inhibitory factor ameliorates ocular Pseudomonas aeruginosa-induced keratitis. PLoS Pathog 6, e1000826 (2010).

22. Niederkorn, J. Y. See no evil, hear no evil, do no evil: the lessons of immune privilege. Nature immunology 7, 354-359 (2006).

23. Kwon, B. \& Hazlett, L. D. Association of CD4+ T cell-dependent keratitis with genetic susceptibility to Pseudomonas aeruginosa ocular infection. J Immunol 159, 6283-6290 (1997)

24. Hazlett, L. D., McClellan, S., Kwon, B., \& Barrett, R. Increased severity of Pseudomonas aeruginosa corneal infection in strains of mice designated as Th1 versus Th2 responsive. Investigative ophthalmology \& visual science 41, 805-810 (2000).

25. Brown, D. R. et al. T helper subset differentiation in the absence of invariant chain. J Exp Med 185, 31-41 (1997).

26. Beswick, E. J. et al. Helicobacter pylori-induced IL-8 production by gastric epithelial cells up-regulates CD74 expression. J Immunol 175, 171-176 (2005).

27. Beswick, E. J. et al. Helicobacter pylori binds to CD74 on gastric epithelial cells and stimulates interleukin-8 production. Infection and immunity $\mathbf{7 3}, 2736-2743$ (2005).

28. Weber, C. et al. Structural determinants of MIF functions in CXCR2-mediated inflammatory and atherogenic leukocyte recruitment. Proc Natl Acad Sci U S A 105, 16278-16283 (2008).

29. Merk, M. et al. The Golgi-associated protein p115 mediates the secretion of macrophage migration inhibitory factor. J Immunol 182, 6896-6906 (2009).

30. Bernhagen, J. et al. Purification, bioactivity, and secondary structure analysis of mouse and human macrophage migration inhibitory factor (MIF). Biochemistry 33, 14144-14155 (1994).

31. Preston, M. J. et al. Rapid and sensitive method for evaluating Pseudomonas aeruginosa virulence factors during corneal infections in mice. Infect Immun 63, 3497-3501 (1995).

32. Gadjeva, M., Wang, Y., \& Horwitz, B. H. NF-kappaB p50 and p65 subunits control intestinal homeostasis. European journal of immunology 37, 2509-2517 (2007).

33. Fichorova, R. N. et al. Biological and technical variables affecting immunoassay recovery of cytokines from human serum and simulated vaginal fluid: a multicenter study. Anal Chem 80 (12), 4741-4751 (2008).

\section{Acknowledgements}

This work was supported by Public Health Service grant 5R21EY019944 - 02 (MG) and EY016144 (GP) from the National Eye Institute.

\section{Author contributions}

TZ and MG prepared figures 1,2,5; RF and MG prepared 3 and 4. RF analyzed the MSD cytokine data; TR purified rMIF and prepared the liposomes; SD maintained the mouse colony and provided help with experiments; MG designed experiments, analyzed data and wrote the manuscript text. All authors (MG, SD, TR, RF, JP, TZ) reviewed the manuscript.

\section{Additional information}

Competing financial interests: The authors declare no competing financial interests.

License: This work is licensed under a Creative Commons

Attribution-NonCommercial-NoDerivative Works 3.0 Unported License. To view a copy of this license, visit http://creativecommons.org/licenses/by-nc-nd/3.0/

How to cite this article: Zaidi, T. et al. CD74 deficiency ameliorates Pseudomonas aeruginosa-induced ocular infection. Sci. Rep. 1, 58; DOI:10.1038/srep00058 (2011). 